\title{
Ivermectin-the Trinity of Efficacy, Safety and Tolerabili- ty in the Global Crusade against the Deadly Pandemic of Covid-19
}

\author{
Mini Review \\ Volume 3 Issue 1- 2022
}

\begin{abstract}
Author Details
Prabhpreet Sethi, Amit Sharma*

National Institute of Tuberculosis and Respiratory Diseases (NITRD), India
\end{abstract}

*Corresponding author

Amit Sharma, Pulmonologist, National Institute of Tuberculosis and Respiratory Diseases (NITRD), New Delhi, India

Article History

Received: January 21, 2022 Accepted: January 24, 2022 Published: February 02, 2022

\begin{abstract}
Mini Review
For the last 2 years, mankind is fighting a momentous battle with an adversary which is both lethal and resilient and is still affecting the health and lives of human beings. The Pandemic of Covid-19 has descended upon us relentlessly and has already claimed more than 50 lacs deaths as per records. It is caused by a Corona virus named SARS$\mathrm{CoV}_{2}$ whose first case was reported in China in December 2019 [1].
\end{abstract}

A lot of research and trials have been done or are still ongoing to find treatment of this deadly virus. The virus generally targets the Upper and Lower Respiratory Systems and can lead to severe Pneumonia followed by Acute Respiratory Distress Syndrome (ARDS) which may further lead to involvement of other Systems of the body and an ultimately death. As per existing records, 276,421,237 people have been infected of whom 5,593,457 (2\%) have died till date [2]. Hence, there is a dire need of finding a drug which may help in the recovery of the affected patients by either targeting the virus replication and/or suppress the excessive immune response to it by the host's body which damages the lungs and other organ systems.

In this short review, we will discuss the drug, Ivermectin, specifically in context with the Covid-19 infection. This is a repurposed drug which means that it was already discovered and was being used to treat other conditions. First discovered in 1975, it finds use as an antiparasitic drug in the treatment of onchocerciasis, scabies, ascariasis, lymphatic filariasis etc [3]. Initially it was considered beneficial in the management of Covid-19 pneumonia but the WHO made a recommendation against using it beyond warned against the use of this drug as it was considered that scientific evidence to favor its utility in Covid-19 was misleading and not useful [4].

However, the trials which had begun in the Covid-19 waves have now started to gradually show a brighter side of the drug. Most of the results even in preliminary stages are showing the effectiveness of Ivermectin in the management of Covid-19. Ivermectin has been available since 1981 and with its quadruple advantages of good efficacy, safety, tolerability and cost-effectiveness, it has been placed on the $21^{\text {st }}$ position of the WHO list of essential medicines. These attributes of this drug hold the promise of banishing this pandemic to the remotest corners of the world $[5,6]$.

A recently concluded study has also demonstrated that Ivermectin is effective not only as an inhibitor of the virus but also has utility in controlling the cytokine storm which wreaks havoc to the human body even though they are produced by the same body [7]

There is emerging data that Ivermectin may also be useful in the prevention of the disease in high risk populations especially healthcare workers which strongly emphasizes the role of this drug in prophylaxis [8].

To conclude, Ivermectin is an oral drug which has shown efficacy in the treatment of Covid-19 patients and prophylaxis to high risk populations which is evident by the results of number of randomized Control Trials. Keeping in mind its efficacy, safety, tolerability and compliance, it may be utilized more by Clinicians in their management protocol of Covid-19 infections.

\section{References}

1. Liu YC, Kuo RL, Shih SR (2020) COVID-19: The first documented coronavirus pandemic in history. Biomed J 43(4): 328-333.

2. https://www.worldometers.info/coronavirus/

3. Ottesen EA, Campbell WC (1994) Ivermectin in human medicine. J Antimicrob Chemother 34(2): 195-203.

4. World Health Organization (2021) Therapeutics and COVID-19: Living Guideline. Geneva, Switzerland. 
5. World Health Organization (2019) World Health Organization model list of essential medicines: $21^{\text {st }}$ list 2019. Geneva: Switzerland.

6. Bryant A, Lawrie TA, Dowswell T, Fordham EJ, Mitchell S, et al. (2021) Ivermectin for Prevention and Treatment of COVID-19 Infection: A Systematic Review, Meta-analysis, and Trial Sequential Analysis to Inform Clinical Guidelines. Am J Ther 28(4): e434-e460.
7. Rezk N, Sileem AE, Gad D, Khalil A (2021) miRNA-223-3p, miRNA2909 and Cytokines Expression in COVID-19 Patients Treated with Ivermectin. Zagazig University Medical Journal.

8. Behera P, Patro BK, Singh AK, Chandanshive PD, Ravikumar S R, et al. (2021) Role of ivermectin in the prevention of SARS-CoV-2 infection among healthcare workers in India: A matched case-control study. PLoS One 16(2): e0247163. 